Algebraic 85 Geometric Topology

Volume 2 (2002) 137-155

Published: 28 February 2002

\title{
A norm for the cohomology of 2-complexes
}

\author{
VLADIMIR TURAEV
}

\begin{abstract}
We introduce a norm on the real 1-cohomology of finite 2complexes determined by the Euler characteristics of graphs on these complexes. We also introduce twisted Alexander-Fox polynomials of groups and show that they give rise to norms on the real 1-cohomology of groups. Our main theorem states that for a finite 2-complex $X$, the norm on $H^{1}(X ; \mathbb{R})$ determined by graphs on $X$ majorates the Alexander-Fox norms derived from $\pi_{1}(X)$.
\end{abstract}

AMS Classification 57M20; 57M05

Keywords Group cohomology, norms, 2-complexes, Alexander-Fox polynomials

\section{Introduction}

We introduce a (possibly degenerate) norm on the real 1-cohomology of finite 2-complexes. The definition of this norm is similar to Thurston's definition of a norm on the 2-homology of 3-manifolds. The key difference is that instead of surfaces in 3-manifolds we consider graphs on 2-complexes. In many instances the resulting theory is similar to but simpler than the one of Thurston.

In generalization of the standard Alexander-Fox polynomial of groups we introduce twisted Alexander-Fox polynomials. We show that they determine norms on the real 1-cohomology of groups.

Our main result is a comparison theorem which states that for a finite 2complex $X$, the norm on $H^{1}(X ; \mathbb{R})$ determined by graphs on $X$ majorates the Alexander-Fox norms derived from $\pi_{1}(X)$.

This result is a cousin of the classical Seifert inequality in knot theory which says that the genus of a knot $K \subset S^{3}$ is greater than or equal to the half of the span of the Alexander polynomial of $K$. A more general estimate from below for the Thurston norm appeared in the Seiberg-Witten theory in dimension 3, see [1], [3], [4], [5]. This estimate is a 3-dimensional version of the much deeper 
adjunction inequality in dimension 4 . A related (weaker) result in dimension 3 appeared also in [6].

We state here a sample application of our main theorem to codimension 1 submanifolds of triangulated manifolds. Let $M$ be a closed connected oriented triangulated manifold of dimension $m \geq 3$. Let $S \subset M$ be a closed oriented $(m-1)$-dimensional submanifold of $M$ representing a non-zero element $s \in$ $H_{m-1}(M ; \mathbb{Z})=H^{1}(M ; \mathbb{Z})$. Let $n$ be the maximal positive integer dividing $s$ in $H^{1}(M ; \mathbb{Z})$. Assume that $S$ intersects the 2-skeleton $M^{(2)}$ transversely along a (finite 1-dimensional) $\mathrm{CW}$-space $\Gamma=S \cap M^{(2)}$. If $\pi_{1}(M)=\pi_{1}\left(S^{3} \backslash K\right)$ where $K$ is a knot in $S^{3}$ then $|\chi(\Gamma)| \geq n(d-1)$ where $d$ is the span of the Alexander polynomial of $K$. For example, if $\pi_{1}(M)=\left\langle x, y: x^{p} y^{q}=1\right\rangle$ is the group of a torus $(p, q)$-knot with relatively prime integers $p, q \geq 2$ then $|\chi(\Gamma)| \geq n(p q-p-q)$.

\section{A norm on the 1-cohomology of a 2-complex}

1.1 Two-complexes By a graph we mean a finite CW-complex of dimension $\leq 1$. By a finite 2-complex we mean the underlying topological space of a finite 2-dimensional $\mathrm{CW}$-complex such that each its point has a neighborhood homeomorphic to the cone over a graph. The latter condition is aimed at eliminating all kinds of local wilderness. Examples of finite 2-complexes: compact surfaces; 2-skeletons of finite simplicial spaces; products of graphs with a closed interval.

We define two subspaces $\operatorname{Int} X$ and $\partial X$ of a finite 2-complex $X$. The subspace Int $X \subset X$ consists of the points which have a neighborhood homeomorphic to $\mathbb{R}^{2}$. Clearly, Int $X$ is a 2-manifold with finite number of components. Its complement $X \backslash \operatorname{Int} X$ is a graph contained in the 1-skeleton of any CW-decomposition of $X$.

The boundary $\partial X$ of $X$ is the closure in $X$ of the set of all points of $X \backslash \operatorname{Int} X$ which have an open neighborhood in $X$ homeomorphic to $\mathbb{R}$ or to $\mathbb{R}_{+}^{2}=$ $\left\{(a, b) \in \mathbb{R}^{2}, b \geq 0\right\}$. A simple local analysis shows that $\partial X$ is a graph contained in the 1-skeleton of any CW-decomposition of $X$. If $X$ is a compact surface then $\partial X$ is its boundary in the usual sense and $\operatorname{Int} X=X \backslash \partial X$.

1.2 Graphs on 2-complexes A graph $\Gamma$ embedded in a finite 2-complex $X$ is regular if $\Gamma \subset X \backslash \partial X$ and there are a closed neighborhood $U$ of $\Gamma$ in $X \backslash \partial X$ and a homeomorphism $U \approx \Gamma \times[-1,1]$ sending any point $x \in \Gamma$ to $x \times 0$. If $\Gamma$ 
is connected then $U \backslash \Gamma$ has two components. A choice of one of them is called a coorientation of $\Gamma$. If $\Gamma$ is not connected then a coorientation of $\Gamma$ is a choice of coorientation for all components of $\Gamma$.

Any vertex of a regular graph $\Gamma \subset X$ is incident to at least two edges of $\Gamma$ (counting with multiplicity). Hence $\chi(\Gamma) \leq 0$. Set $\chi_{-}(\Gamma)=-\chi(\Gamma) \geq 0$.

A cooriented regular graph $\Gamma \subset X$ determines a 1-dimensional cohomology class $s_{\Gamma} \in H^{1}(X, \partial X)=H^{1}(X, \partial X ; \mathbb{Z})$ as follows. Choose a neighborhood $U$ of $\Gamma$ and a homeomorphism $f: U \rightarrow \Gamma \times[-1,1]$ as above so that the coorientation of $\Gamma$ is determined by the components of $U \backslash \Gamma$ lying in $f^{-1}(\Gamma \times(0,1])$. We define a map $g: X / \partial X \rightarrow S^{1}=\{z \in \mathbb{C},|z|=1\}$ by $g(X \backslash U)=-1 \in S^{1}$ and $g(x)=\exp (\pi i \tilde{f}(x))$ for $x \in U$ where $\tilde{f}(x) \in[-1,1]$ is the projection of $f(x) \in \Gamma \times[-1,1]$ to $[-1,1]$. Set $s_{\Gamma}=g^{*}\left(s_{0}\right)$ where $s_{0}$ is the generator of $H^{1}\left(S^{1}\right)=\mathbb{Z}$ determined by the counterclockwise orientation of $S^{1}$. It is clear that $s_{\Gamma}$ does not depend on the choice of $U$ and $f$. To evaluate $s_{\Gamma}$ on the homology class of a path in $X$ whose endpoints either coincide or lie in $\partial X$, one should count the algebraic number of intersections of this path with $\Gamma$. If $\Gamma=\emptyset$, then $s_{\Gamma}=0$.

A simple transversality argument shows that for any $s \in H^{1}(X, \partial X)$ there is a cooriented regular graph $\Gamma \subset X$ such that $s=s_{\Gamma}$. It can be constructed as follows. First, one realises $s$ as $g^{*}\left(s_{0}\right)$ for a certain map $g: X \rightarrow S^{1}$ sending $\partial X$ to $-1 \in S^{1}$. Secondly, one fixes a CW-decomposition of $X$ and deforms $g$ so that it maps the 0 -skeleton $X^{(0)}$ of $X$ into $S^{1} \backslash\{1\}$. Then one deforms $g\left(\operatorname{rel} X^{(0)}\right)$ so that its restriction to the 1-skeleton $X^{(1)}$ of $X$ becomes transversal to the point $1 \in S^{1}$. Finally, one deforms $g\left(\operatorname{rel} X^{(1)}\right)$ so that its restriction to any 2-cell of $X$ becomes transversal to $1 \in S^{1}$. Then $\Gamma=g^{-1}(1)$ is a regular graph on $X$ and $g$ determines its coorientation such that $s_{\Gamma}=s$.

1.3 A norm on $H^{1}(X, \partial X ; \mathbb{R})$ By a norm on a real vector space $V$ we mean an $\mathbb{R}$-valued function $\|\ldots\|$ on $V$ such that $\|s\| \geq 0$ and $\left\|s+s^{\prime}\right\| \leq\|s\|+\left\|s^{\prime}\right\|$ for any $s, s^{\prime} \in V$. A norm is allowed to be degenerate, i.e., to vanish on nonzero vectors. A norm $\|\ldots\|$ on $V$ is homogeneous, if $\|k s\|=|k|\|s\|$ for any $k \in \mathbb{R}, s \in V$. One similarly defines norms on lattices, the only difference is that in the definition of homogeneity $k \in \mathbb{Z}$.

Let $X$ be a finite 2-complex. For $s \in H^{1}(X, \partial X)=H^{1}(X, \partial X ; \mathbb{Z})$, set

$$
\|s\|=\min _{\Gamma, s_{\Gamma}=s} \chi_{-}(\Gamma)
$$

where $\Gamma$ runs over cooriented regular graphs in $X$ such that $s=s_{\Gamma}$. The next lemma shows that $\|\ldots\|$ is a homogeneous norm on $H^{1}(X, \partial X)$. It extends 
uniquely to a homogeneous continuous norm on $H^{1}(X, \partial X ; \mathbb{R})$ denoted $\|\ldots\|_{X}$ or simply $\|\ldots\|$.

1.4 Lemma $\|\ldots\|$ is a homogeneous norm on $H^{1}(X, \partial X)$.

Proof We verify that $\left\|s+s^{\prime}\right\| \leq\|s\|+\left\|s^{\prime}\right\|$ for any $s, s^{\prime} \in H^{1}(X, \partial X)$. Let $\Gamma, \Gamma^{\prime}$ be cooriented regular graphs in $X$ such that $s=s_{\Gamma}, s^{\prime}=s_{\Gamma^{\prime}}$. We slightly deform $\Gamma$ so that $\Gamma \cap \Gamma^{\prime} \subset \operatorname{Int} X$ and each point $\gamma \in \Gamma \cap \Gamma^{\prime}$ is a transversal intersection of an (open) edge of $\Gamma$ with an (open) edge of $\Gamma^{\prime}$. A smoothing of $\Gamma \cup \Gamma^{\prime}$ at $\gamma$ replaces the crossing at $\gamma$ by the $\supset \subset$-type configuration. There is a unique smoothing at $\gamma$ such that the coorientations of $\Gamma, \Gamma^{\prime}$ induce (locally) a coorientation of the resulting graph. Applying this smoothing at all points of $\Gamma \cap \Gamma^{\prime}$ we transform $\Gamma \cup \Gamma^{\prime}$ into a cooriented regular graph, $\Gamma^{\prime \prime}$, in $X$. It is obvious that $s_{\Gamma^{\prime \prime}}=s+s^{\prime}$ and $\left\|s_{\Gamma^{\prime \prime}}\right\| \leq \chi_{-}\left(\Gamma^{\prime \prime}\right)=\chi_{-}(\Gamma)+\chi_{-}\left(\Gamma^{\prime}\right)$. Therefore $\left\|s+s^{\prime}\right\| \leq\|s\|+\left\|s^{\prime}\right\|$.

The homogeneity of $\|\ldots\|$ is proven by the same argument as in [7], p.103. The key point is that if a cooriented regular graph $\Gamma$ in $X$ represents $k s$ with integer $k \geq 1$ and $s \in H^{1}(X, \partial X)$ then $\Gamma$ splits as a disjoint union of $k$ graphs representing $s$. This implies that $\|k s\| \geq k\|s\|$. The opposite inequality is obvious since for any $\Gamma \subset X$ representing $s$ a union of $k$ parallel copies of $\Gamma$ represents $k s$.

1.5 Properties of $\|\ldots\|_{X}$ (1) Replacing everywhere embedded graphs in $X$ by immersed graphs, we obtain the same norm. (By an immersed graph we mean a graph in $X$ which locally looks like an embedded graph or like a transversal crossing of two embedded $\operatorname{arcs}$ in $\operatorname{Int} X$.) The immersed graphs lead to the same norm because the smoothing of an immersed graph at all its double points yields an embedded graph with the same Euler characteristic.

(2) It is easy to describe the subset of $H^{1}(X, \partial X)$ consisting of the vectors with zero norm. Indeed, for a regular graph $\Gamma \subset X$ we have $\chi(\Gamma)=0$ if and only if $\Gamma$ is a closed 1-dimensional submanifold of $\operatorname{Int} X$. Therefore the set of vectors in $H^{1}(X, \partial X)$ with zero norm coincides with the set of vectors $s_{\Gamma}$ corresponding to cooriented closed 1-manifolds $\Gamma \subset \operatorname{Int} X$. The argument in [7], p.105 shows that the set of vectors in $H^{1}(X, \partial X ; \mathbb{R})$ with zero norm is the $\mathbb{R}$-linear span of such $s_{\Gamma}$.

(3) If all components of Int $X$ are open 2-discs or Möbius bands then the norm $\|s\|_{X}$ vanishes only for $s=0$. The unit ball $\left\{s \in H^{1}(X, \partial X ; \mathbb{R}),\|s\| \leq 1\right\}$ is then a compact convex polytope symmetric in the origin. It can be defined 
by a system of inequalities $|\beta(s)| \leq 1$ where $\beta$ runs over a finite subset of $H_{1}(X, \partial X)$. This follows from general properties of norms taking integral values on a lattice of maximal rank, see [7], p. 106.

(4) If $p: \tilde{X} \rightarrow X$ is an $n$-sheeted covering with $n \geq 2$ then $\partial \tilde{X}=p^{-1}(\partial X)$ and for any $s \in H^{1}(X, \partial X ; \mathbb{R})$, we have $\left\|p^{*}(s)\right\|=n\|s\|$ where $p^{*}$ is the induced homomorphism $H^{1}(X, \partial X ; \mathbb{R}) \rightarrow H^{1}(\tilde{X}, \partial \tilde{X} ; \mathbb{R})$. Indeed, if $\Gamma$ is a cooriented regular graph in $X$ representing $s$ then the graph $p^{-1}(\Gamma) \subset \tilde{X}$ represents $p^{*}(s)$. Therefore $\left\|p^{*}(s)\right\| \leq n\|s\|$. On the other hand, if $\Gamma^{\prime}$ is a cooriented regular graph in $\tilde{X}$ representing $p^{*}(s)$ then deforming if necessary $\Gamma^{\prime}$ we can assume that $p\left(\Gamma^{\prime}\right)$ is an immersed graph. Smoothing it at all crossing points we obtain a cooriented regular graph $\Gamma \subset X$ such that $\chi(\Gamma)=\chi\left(\Gamma^{\prime}\right)$ and $s_{\Gamma}=n s$. Hence $n\|s\|=\|n s\| \leq\left\|p^{*}(s)\right\|$.

1.6 A computation from cocycles For any finite 2-complex $X$ and $s \in$ $H^{1}(X, \partial X)$, we can compute $\|s\|_{X}$ in terms of 1 -cocycles on $X$. Fix a CWdecomposition of $X$ and orient all its edges (= open 1-cells). Consider a $\mathbb{Z}$ valued cellular 1-cocycle $k$ on $(X, \partial X)$. Set $|k|=\sum_{e}\left(n_{e} / 2-1\right)|k(e)|$ where $e$ runs over all edges of $X$ not lying on $\partial X, n_{e} \geq 2$ is the number of 2-cells of $X$ adjacent to $e$ (counted with multiplicity), and $k(e) \in \mathbb{Z}$ is the value of $k$ on $e$. We claim that $\|s\|_{X}=\min _{k}|k|$ where $k$ runs over all cellular 1-cocycles on $(X, \partial X)$ representing $s$. This reduces the computation of $\|s\|$ to a standard algorithmically solvable minimization problem on a lattice.

We first prove that $\|s\| \leq|k|$ for any $k$ as above. Choose $|k(e)|$ distinct points on each edge $e$ of $X$ not lying on $\partial X$. Provide these points with positive coorientation on $e$ if $k(e)>0$ and with negative coorientation on $e$ if $k(e)<0$ (recall that $e$ is oriented). The boundary of each 2-cell of $X$ meets a certain number of these distinguished points. By the cocycle condition, their algebraic number is 0 so that we can join these points in the 2-cell by disjoint cooriented intervals compatible with the coorientation at the endpoints. Proceeding in this way in all 2-cells of $X$ we obtain a cooriented regular graph $\Gamma \subset X$. It is clear that $\Gamma$ represents $s$. Therefore $\|s\| \leq \chi_{-}(\Gamma)=-\chi(\Gamma)=|k|$. Conversely, any cooriented regular graph $\Gamma \subset X \backslash X^{(0)}$ representing $s$ defines a $\mathbb{Z}$-valued 1-cocycle $k$ on $(X, \partial X)$ whose value on an (oriented) edge $e$ is equal to the intersection number $e \cdot \Gamma$. This cocycle represents $s$ and an easy computation shows that $\chi_{-}(\Gamma)=-\chi(\Gamma) \geq|k|$. Therefore $\|s\| \geq \min _{k}|k|$.

The formula $\|s\|=\min _{k}|k|$ is especially useful in the cases where either all 0-cells of $X$ lie on $\partial X$ or $\partial X=\emptyset$ and $X$ has only one 0-cell. In both cases every cohomology class $s \in H^{1}(X, \partial X)$ is represented by a unique cocycle. 
1.7 Examples (1) If $X$ is a compact surface then all elements of $H^{1}(X, \partial X)$ are represented by regular graphs consisting of disjoint embedded circles. Therefore the norm $\|\ldots\|_{X}$ on $H^{1}(X, \partial X ; \mathbb{R})$ vanishes.

(2) Let $\Gamma$ be a graph such that all its vertices are incident to at least two edges (counted with multiplicity). Let $f$ be a homeomorphism of $\Gamma$ onto itself. The mapping torus, $X$, of $f$ is a 2-complex with void boundary. The fibers of the natural fibration $X \rightarrow S^{1}$ determine a class, $s \in H^{1}(X)$. Clearly, $\|s\| \leq \chi_{-}(\Gamma)$. We show in Sect. 3 that $\|s\|=\chi_{-}(\Gamma)$. This example can be generalised to maps $\Gamma \rightarrow \Gamma$ whose mapping torus is a 2 -complex.

(3) Let $\Gamma$ be a graph as in the previous example. The cylinder $X=\Gamma \times[-1,1]$ is a finite 2-complex with $\partial X=\Gamma \times\{-1,1\}$. The graph $\Gamma \times 0 \subset X$ endowed with a coorientation represents a certain $s \in H^{1}(X, \partial X)$. The cylinder $X$ embeds in $\Gamma \times S^{1}$ in the obvious way and therefore it follows from the previous example that $\|s\|=\chi_{-}(\Gamma)$.

1.8 Two-complexes associated with group presentations Let $\pi$ be a group presented by a finite number of generators and relations $\left\langle x_{1}, \ldots, x_{m}\right.$ : $\left.r_{1}, \ldots, r_{n}\right\rangle$ where $r_{1}, \ldots, r_{n}$ are words in the alphabet $x_{1}^{ \pm 1}, \ldots, x_{m}^{ \pm 1}$. In this subsection we consider only presentations such that each generator appears in the relations at least twice. The presentation $\left\langle x_{1}, \ldots, x_{m}: r_{1}, \ldots, r_{n}\right\rangle$ gives rise in the usual way to a 2-dimensional CW-complex $X$ with one 0 -cell, $m$ one-cells and $n$ two-cells. Let $\#\left(x_{i}\right)$ be the total number of appearances of $x_{i}$ in the words $r_{1}, \ldots, r_{n}$. (A power $x_{i}^{k}$ appearing in these words contributes $|k|$ to $\#\left(x_{i}\right)$ ). It is clear that $\#\left(x_{i}\right)$ is the number of 2-cells of $X$ adjacent to the $i$-th 1-cell of $X$. By assumption, $\#\left(x_{i}\right) \geq 2$ for all $i$ so that $\partial X=\emptyset$. Using the formulas of Sect. 1.6, we can compute the norm $\|\ldots\|_{X}$ on $H^{1}(X ; \mathbb{R})=H^{1}(\pi ; \mathbb{R})$ by $\|s\|_{X}=\sum_{i=1}^{n}\left(\#\left(x_{i}\right) / 2-1\right)\left|s\left(x_{i}\right)\right|$ for any $s \in H^{1}(\pi ; \mathbb{R})$. This norm depends on the presentation of $\pi$. It is easy to increase this norm for instance by adding a tautological relation $x_{i} x_{i}^{-1}=1$.

We say that a finite presentation of $\pi$ by generators and relations is minimal if the corresponding norm on $H^{1}(\pi ; \mathbb{R})$ (considered as a function) is smaller than or equal to the norm on $H^{1}(\pi ; \mathbb{R})$ determined by any other finite presentation of $\pi$. For instance, if each generator appears in the relations exactly twice, then the corresponding norm is zero and the group presentation is minimal. Another example: $\pi=\left\langle x, y: x^{p} y^{q}=1\right\rangle$ where $p, q \geq 2$ are relatively prime integers. A generator $s \in H^{1}(\pi)=\mathbb{Z}$ takes values $-q$ and $p$ on $x, y$, respectively. The norm of $s$ with respect to this presentation equals $(p / 2-1) q+(q / 2-1) p=p q-p-q$. We shall show in Sect. 3 that this presentation is minimal. 
1.9 A related construction We describe a related construction which derives a norm on the cohomology of a compact surface $\Sigma$ from a family of loops on $\Sigma$. Let $\alpha=\left\{\alpha_{i}\right\}_{i}$ be a finite family of closed curves in $\operatorname{Int} \Sigma$ whose all crossings and self-crossings are transversal double intersections. We define a norm $|\ldots|_{\alpha}$ on $H^{1}(\Sigma, \partial \Sigma ; \mathbb{R})$ as follows. For any $s \in H^{1}(\Sigma, \partial \Sigma)$, set $|s|_{\alpha}=\min _{S} \#\left(S \cap \cup_{i} \alpha_{i}\right)$ where $S$ runs over cooriented closed 1-dimensional submanifolds of $\Sigma$ representing $s$ and meeting $\cup_{i} \alpha_{i}$ transversely (in the complement of the set of double points of $\left.\cup_{i} \alpha_{i}\right)$. Here $\#\left(S \cap \cup_{i} \alpha_{i}\right)$ is the number of points in $S \cap \cup_{i} \alpha_{i}$. It is easy to check that $|\ldots|_{\alpha}$ is a homogeneous norm on $H^{1}(\Sigma, \partial \Sigma)$. As usual it extends uniquely to a homogeneous continuous norm, also denoted $|\ldots|_{\alpha}$, on $H^{1}(\Sigma, \partial \Sigma ; \mathbb{R})$. This norm is preserved under the first and third Reidemeister moves on the loops $\left\{\alpha_{i}\right\}_{i}$ but in general is not preserved under the second Reidemeister move. A simple example is provided by a small loop $\alpha \subset \Sigma$ bounding a disc in $\Sigma$. The norm $|\ldots|_{\alpha}$ on $H^{1}(\Sigma, \partial \Sigma ; \mathbb{R})$ is zero. On the other hand we can deform $\alpha$ into an immersed loop $\beta$ in $S$ which splits $\Sigma$ into 2-discs. The norm $|\ldots|_{\beta}$ is then non-degenerate.

The norm $|\ldots|_{\alpha}$ on $H^{1}(\Sigma, \partial \Sigma ; \mathbb{R})$ is related to the norm on the 1-cohomology of 2-complexes as follows. Let $\alpha=\left\{\alpha_{i}\right\}_{i}$ be a finite family of loops in $\operatorname{Int} \Sigma$ as above. Let $X$ be the 2-complex obtained by gluing 2-discs to $\Sigma$ along these loops. It is clear that $\partial X=\partial \Sigma$. We can identify $H^{1}(X, \partial X ; \mathbb{R})$ with the linear subspace of $H^{1}(\Sigma, \partial \Sigma ; \mathbb{R})$ consisting of cohomology classes whose evaluation on the loops $\left\{\alpha_{i}\right\}_{i}$ is 0 . Then the norm $\|\ldots\|_{X}$ on $H^{1}(X, \partial X ; \mathbb{R})$ is the restriction of $(1 / 2)|\ldots|_{\alpha}$. Indeed, any regular graph $\Gamma \subset X$ consists of a closed 1-manifold $S=\Gamma \cap \Sigma$ and several intervals lying in the glued 2-discs and connecting the points of $S \cap \cup_{i} \alpha_{i}$. All vertices of $\Gamma$ are trivalent and therefore $\chi_{-}(\Gamma)=-\chi(\Gamma)=(1 / 2) \#\left(S \cap \cup_{i} \alpha_{i}\right)$. The 2-complexes obtained in this way from homotopic systems of loops are (simply) homotopy equivalent. The example above implies that the norm $\|\ldots\|$ in general is not preserved under (simple) homotopy equivalences of 2-complexes.

\section{The Alexander-Fox polynomials and norms}

The Alexander polynomial is mostly known in the context of knot theory. Fox observed that this polynomial depends only on the knot group and in fact can be defined for an arbitrary finitely generated group. In this section we recall the relevant definitions following [2]. In generalisation of the standard AlexanderFox polynomial, we introduce twisted Alexander-Fox polynomials and consider the associated norms on 1-cohomology of groups. 
Fix throughout this section a finitely generated group $\pi$. Set $H=H_{1}(\pi)$ and $G=H /$ Tors $H$. The ring homomorphism $\mathbb{Z}[H] \rightarrow \mathbb{Z}[G]$ induced by the projection $H \rightarrow G$ will be denoted by pr.

2.1 The elementary ideals The group $\pi$ determines an increasing sequence of ideals $E_{0}(\pi) \subset E_{1}(\pi) \subset E_{2}(\pi) \subset \ldots$ of the group ring $\mathbb{Z}[H]$ called the elementary ideals of $\pi$. They can be computed from an arbitrary presentation of $\pi$ by generators and relations $\left\langle x_{1}, \ldots, x_{m}: r_{1}, r_{2}, \ldots\right\rangle$ with finite $m \geq 1$. Here each $r_{i}$ is viewed as an element of the free group, $F$, generated by $x_{1}, \ldots, x_{m}$; the number of relations can be infinite. Every $f \in F$ can be uniquely expanded in $\mathbb{Z}[F]$ as $1+\sum_{j=1}^{m} f_{j}\left(x_{j}-1\right)$ with $f_{1}, \ldots, f_{m} \in \mathbb{Z}[F]$. The element $f_{j} \in \mathbb{Z}[F]$ is called the $j$-th Fox derivative of $f$ and denoted by $\partial f / \partial x_{j}$. Consider the matrix $\left[\partial r_{i} / \partial x_{j}\right]_{i, j}$ over $\mathbb{Z}[F]$. Applying the natural projections $\mathbb{Z}[F] \rightarrow \mathbb{Z}[\pi] \rightarrow \mathbb{Z}[H]$ to the entries of this matrix we obtain a matrix, $A$, over $\mathbb{Z}[H]$ called the Alexander-Fox matrix of the presentation $\left\langle x_{1}, \ldots, x_{m}: r_{1}, r_{2}, \ldots\right\rangle$. It has $m$ columns and possibly infinite number of rows. Adding if necessary to $r_{1}, r_{2}, \ldots$ several copies of the neutral element $1 \in F$ we can assume that $A$ has at least $m$ rows. For $d=0,1, \ldots$, the ideal $E_{d}(\pi) \subset \mathbb{Z}[H]$ is generated by the minor determinants of $A$ of order $m-d$. This ideal does not depend on the presentation of $\pi$. We shall be interested only in the ideal $E_{1}(\pi)$ which will be denoted $E(\pi)$.

2.2 The Alexander-Fox polynomials Consider the ideal $\operatorname{pr}(E(\pi)) \subset$ $\mathbb{Z}[G]$. Since $\mathbb{Z}[G]$ is a unique factorization domain, one can consider the greatest common divisor of the elements of $\operatorname{pr}(E(\pi))$. This gcd is an element of $\mathbb{Z}[G]$ defined up to multiplication by $\pm G$. It is called the Alexander-Fox polynomial of $\pi$ and denoted $\Delta(\pi)$.

The obvious inclusion $\operatorname{pr}(E(\pi)) \subset \Delta(\pi) \mathbb{Z}[G]$ can be slightly improved provided rk $H \geq 2$. Namely, if rk $H \geq 2$, then

$$
\operatorname{pr}(E(\pi)) \subset \Delta(\pi) J
$$

where $J$ is the augmentation ideal of $\mathbb{Z}[G]$. This inclusion goes back to $[2]$, Prop. 6.4 at least in the case Tors $H=0$. We give a proof of (2.a) at the end of Sect. 2.

In generalisation of $\Delta(\pi)$, we define twisted Alexander-Fox polynomials of $\pi$ numerated by $\sigma \in(\operatorname{Tors} H)^{*}=\operatorname{Hom}\left(\operatorname{Tors} H, \mathbb{C}^{*}\right)$. Fix a splitting $H=\operatorname{Tors} H \times$ $G$. For $\sigma \in(\text { Tors } H)^{*}$, consider the ring homomorphism $\tilde{\sigma}: \mathbb{Z}[H] \rightarrow \mathbb{C}[G]$ sending $f g$ with $f \in$ Tors $H, g \in G$ to $\sigma(f) g$ where $\sigma(f) \in \mathbb{C}^{*} \subset \mathbb{C}$. The ring $\mathbb{C}[G]$ is a unique factorization domain and we can set $\Delta^{\sigma}(\pi)=\operatorname{gcd} \tilde{\sigma}(E(\pi))$. 
This gcd is an element of $\mathbb{C}[G]$ defined up to multiplication by elements of $G$ and nonzero complex numbers. Under a different choice of the splitting $H=\operatorname{Tors} H \times G$, the polynomial $\Delta^{\sigma}(\pi)$, represented say by $\sum_{g \in G} c_{g} g$ with $c_{g} \in \mathbb{C}$, is replaced by $\sum_{g \in G} c_{g} \sigma(\psi(g)) g$ where $\psi \in \operatorname{Hom}(G$, Tors $H)$. For $\sigma=1$, we have $\Delta^{1}(\pi)=\mathbb{C}^{*} \Delta(\pi)$.

2.3 The Alexander-Fox polytopes and norms Fix $\sigma \in(\operatorname{Tors} H)^{*}$. In analogy with the Newton polytope of a polynomial, we can derive from $\Delta^{\sigma}(\pi)$ an Alexander-Fox polytope (or briefly AF-polytope) $P^{\sigma}(\pi) \subset H_{1}(\pi ; \mathbb{R})$. Pick a representative $\sum_{g \in G} c_{g} g \in \mathbb{C}[G]$ of $\Delta^{\sigma}(\pi)$. Set

$$
P^{\sigma}(\pi)=H U L L\left(\left\{\frac{1}{2}\left(g^{r e a l}-\left(g^{\prime}\right)^{r e a l}\right) \mid g, g^{\prime} \in G, \quad c_{g} \neq 0, c_{g^{\prime}} \neq 0\right\}\right)
$$

where $g^{\text {real }} \in H_{1}(\pi ; \mathbb{R})$ is the real homology class represented by $g \in G$ and for a subset $S$ of a linear space, $H U L L(S)$ denotes the convex hull of $S$. By convention, if $\Delta^{\sigma}(\pi)=0$ then $P^{\sigma}(\pi)=\{0\}$. The polytope $P^{\sigma}(\pi)$ is a compact convex polytope symmetric in the origin and independent of the representative $\sum_{g} c_{g} g$. Its vertices lie on the half-integral lattice $(1 / 2) G$ where $G \subset H_{1}(\pi ; \mathbb{R})$ consists of integral homology classes.

We define the Alexander-Fox norm (or briefly AF-norm) $\|\ldots\|^{\sigma}$ on $H^{1}(\pi ; \mathbb{R})$ by

$$
\|s\|^{\sigma}=2 \max _{x \in P^{\sigma}(\pi)}|s(x)|=\max _{g, g^{\prime} \in G, c_{g} c_{g^{\prime}} \neq 0}\left|s(g)-s\left(g^{\prime}\right)\right|
$$

where $s \in H^{1}(\pi ; \mathbb{R})$ and $s(x) \in \mathbb{R}$ is the evaluation of $s$ on $x$. This norm is continuous and homogeneous. It was first considered in the case $\sigma=1$ by C. McMullen [6].

The AF-norms are natural with respect to group isomorphisms: For a group isomorphism $\varphi: \pi^{\prime} \rightarrow \pi$ and $s \in H^{1}(\pi ; \mathbb{R}), \sigma \in\left(\operatorname{Tors} H_{1}(\pi)\right)^{*}$, we have $\|s\|^{\sigma}=$ $\left\|\varphi^{*}(s)\right\|^{\sigma \varphi_{*}}$ where $\varphi^{*}$ and $\varphi_{*}$ are the induced homomorphisms $H^{1}(\pi ; \mathbb{R}) \rightarrow$ $H^{1}\left(\pi^{\prime} ; \mathbb{R}\right)$ and Tors $H_{1}\left(\pi^{\prime}\right) \rightarrow \operatorname{Tors} H_{1}(\pi)$, respectively.

2.4 Examples (1) If $\pi$ has a presentation with $m$ generators and $\leq m-2$ relations then $E(\pi)=0$ and the AF-norms on $H^{1}(\pi ; \mathbb{R})$ are 0 .

(2) Let $p, q \geq 2$ be relatively prime integers and $\pi=\left\langle x, y: x^{p} y^{q}=1\right\rangle$. Let $t$ be a generator of $H_{1}(\pi)=\mathbb{Z}$. Set $n=p q-p-q+1$. The polynomial $\Delta(\pi)$ is represented by the Laurent polynomial $\left(t^{p q}-1\right)(t-1)\left(t^{p}-1\right)^{-1}\left(t^{q}-1\right)^{-1}$ with lowest term 1 and highest term $t^{n}$. The AF-polytope in $H_{1}(\pi ; \mathbb{R})=\mathbb{R}$ is the interval with endpoints $-(n / 2) t^{\text {real }}$ and $(n / 2) t^{\text {real }}$. The AF-norm of both generators of $H^{1}(\pi)=\mathbb{Z}$ is equal to $n=p q-p-q+1$. 
(3) Let $\pi=\left\langle x, y: x^{k} y^{l} x^{-k} y^{-l}=1, y^{m}=1\right\rangle$ where $k, l \geq 1, m \geq 2$. It is clear that $H_{1}(\pi)=\mathbb{Z} \times(\mathbb{Z} / m \mathbb{Z})$ with generators $[x],[y]$ represented by $x, y$. A direct computation shows $E(\pi)$ is generated by 3 elements: $1+[y]+\ldots+$ $[y]^{m-1},\left(1+[x]+\ldots+[x]^{k-1}\right)\left([y]^{l}-1\right)$, and $\left([x]^{k}-1\right)\left(1+[y]+\ldots+[y]^{l-1}\right)$. Setting $[y]=1$ we obtain that $\Delta(\pi)=\operatorname{gcd}(l, m)$. The corresponding AF-norm is zero. Let $\sigma$ be a nontrivial character of Tors $H_{1}(\pi)=\mathbb{Z} / m \mathbb{Z}$. Then $\zeta=\sigma([y]) \neq 1$ is a complex root of unity of order $m$. If $\zeta^{l}=1$ then $\Delta^{\sigma}(\pi)=0$ and the corresponding AF-norm is zero. If $\zeta^{l} \neq 1$ then $\Delta^{\sigma}(\pi)=1+t+\ldots+t^{k-1}$ where $t$ is the generator of $H_{1}(\pi) / \operatorname{Tors} H_{1}(\pi)$ represented by $x$. The corresponding AF-norm of both generators of $H^{1}(\pi)=\mathbb{Z}$ is equal to $k-1$. This example shows that the twisted AF-polynomials may provide more interesting norms than the untwisted AF-polynomial.

2.5 Remark The structure of the ideal $E(\pi)$ can be sometimes described using the theory of Reidemeister torsions. Suppose that $\pi=\pi_{1}(X)$ where $X$ is a finite connected 2-complex with $\chi(X)=0$. As above, set $H=H_{1}(X)=$ $H_{1}(\pi), G=H / \operatorname{Tors} H$. The maximal abelian torsion $\tau(X)$ is an element of the commutative ring $Q(H)$ obtained from $\mathbb{Z}[H]$ by inverting all non-zerodivisors (see [8],[9]). The natural homomorphism $\mathbb{Z}[H] \rightarrow Q(H)$ is an inclusion and we can identify $\mathbb{Z}[H]$ with its image. Then $E(\pi)=\tau(X) I$ where $I$ is the augmentation ideal of $\mathbb{Z}[H]$ (for a proof, see [8], p. 689). If $\operatorname{rk} H \geq 2$, then $\tau(X) \in \mathbb{Z}[H]$ and for any $\sigma \in(\text { Tors } H)^{*}$, the twisted AF-polynomial $\Delta^{\sigma}(\pi)$ is represented by $\tilde{\sigma}(\tau(X)) \in \mathbb{C}[G]$. If $\operatorname{rk} H=1$, then $\tau(X)$ splits as a sum $a+(t-1)^{-1} \Sigma$ where $a \in \mathbb{Z}[H], \Sigma=\sum_{f \in \operatorname{Tors} H} f \in \mathbb{Z}[H]$, and $t$ is any element of $H$ whose projection $\operatorname{pr}(t) \in G=\mathbb{Z}$ is a generator. Then for any non-trivial character $\sigma \in(\text { Tors } H)^{*}$, the polynomial $\Delta^{\sigma}(\pi)$ is represented by $\tilde{\sigma}(a) \in \mathbb{C}[G]$. The polynomial $\Delta(\pi)$ corresponding to $\sigma=1$ is represented by $\operatorname{pr}((t-1) a)+$ $\mid$ Tors $H \mid \in \mathbb{Z}[G]$.

2.6 Proof of (2.a) Consider a presentation $\left\langle x_{1}, \ldots, x_{m}: r_{1}, r_{2}, \ldots\right\rangle$ of $\pi$ by generators and relations with finite $m \geq 1$ and at least $m$ relations. Let $A$ be the Alexander matrix of this presentation. It is enough to show that for any minor determinant $D$ of $A$ of order $m-1$, we have $\operatorname{pr}(D) \in \Delta(\pi) J$. Assume for concreteness that $D$ is the determinant of a submatrix of the first $m-1$ rows of $A$. Let $\pi^{\prime}$ be the group $\left\langle x_{1}, \ldots, x_{m}: r_{1}, r_{2}, \ldots, r_{m-1}\right\rangle$. Set $H^{\prime}=H_{1}\left(\pi^{\prime}\right)$. The natural surjection $H^{\prime} \rightarrow H=H_{1}(\pi)$ induces a ring homomorphism $\mathbb{Z}\left[H^{\prime}\right] \rightarrow$ $\mathbb{Z}[H]$ denoted $\psi$. It follows from definitions that $D \in \psi\left(E\left(\pi^{\prime}\right)\right) \subset E(\pi)$. Note that $\operatorname{rk} H^{\prime} \geq \operatorname{rk} H \geq 2$.

Consider the 2-dimensional CW-complex $X$ determined by the presentation $\left\langle x_{1}, \ldots, x_{m}: r_{1}, r_{2}, \ldots, r_{m-1}\right\rangle$. Clearly, $\pi_{1}(X)=\pi^{\prime}$ and $\chi(X)=0$. By Remark 
2.5, $E\left(\pi^{\prime}\right)=\tau I^{\prime}$ where $\tau \in \mathbb{Z}\left[H^{\prime}\right]$ and $I^{\prime}$ is the augmentation ideal of $\mathbb{Z}\left[H^{\prime}\right]$. Applying pr $\circ \psi$ we obtain that

$$
(\operatorname{pr} \circ \psi)(\tau) J=(\operatorname{pr} \circ \psi)\left(\tau I^{\prime}\right)=(\operatorname{pr} \circ \psi)\left(E\left(\pi^{\prime}\right)\right) \subset \operatorname{pr}(E(\pi)) \subset \Delta(\pi) \mathbb{Z}[G] .
$$

Since $\operatorname{rk} H \geq 2$, we have gcd $J=1$ and hence $\Delta(\pi)$ is a divisor of $(\operatorname{pr} \circ \psi)(\tau) \in$ $\mathbb{Z}[G]$. Therefore $\operatorname{pr}(D) \in(\operatorname{pr} \circ \psi)\left(E\left(\pi^{\prime}\right)\right)=(\operatorname{pr} \circ \psi)(\tau) J \subset \Delta(\pi) J$.

\section{Main theorem}

To state our main theorem it is convenient to introduce a trivial norm $|\ldots|_{0}$ on the real 1-cohomology $H^{1}(X ; \mathbb{R})$ of any $\mathrm{CW}$-space $X$. If the first Betti number of $X$ is $\neq 1$ then $|s|_{0}=0$ for all $s \in H^{1}(X ; \mathbb{R})$. If the first Betti number of $X$ is 1 , then $|\ldots|_{0}$ is the unique homogeneous norm on $H^{1}(X ; \mathbb{R})$ taking value 1 on both generators of $\mathbb{Z}=H^{1}(X ; \mathbb{Z}) \subset H^{1}(X ; \mathbb{R})$.

3.1 Theorem Let $X$ be a connected finite 2-complex with $\partial X=\emptyset$. For any $s \in H^{1}(X ; \mathbb{R})$ and any $\sigma \in\left(\operatorname{Tors} H_{1}(X)\right)^{*}$,

$$
\|s\|_{X} \geq\|s\|^{\sigma}-\delta_{\sigma}^{1}|s|_{0}
$$

where $\|\ldots\|^{\sigma}$ is the Alexander-Fox norm on $H^{1}(X ; \mathbb{R})=H^{1}\left(\pi_{1}(X) ; \mathbb{R}\right)$ determined by $\sigma$ and $\delta_{\sigma}^{1}=1$ if $\sigma=1$ and $\delta_{\sigma}^{1}=0$ otherwise.

Theorem 3.1 will be proven in Sect. 4. Note that the norm $\|\ldots\|^{\sigma}$ on $H^{1}(X ; \mathbb{R})$ does not depend on the choice of a base point in $X$ because of the invariance of the AF-norms under group isomorphisms. In the case $\operatorname{rk} H_{1}(X) \geq 2$, (3.a) simplifies to $\|s\|_{X} \geq\|s\|^{\sigma}$.

Inequality (3.a) has a version for 1-cohomology classes on 3-manifolds, where on the left hand side appears the Thurston norm of $s$ and the right hand side is $\|\left. s\right|^{\sigma}-2 \delta_{\sigma}^{1}|s|_{0}$. The author plans to discuss this version of Theorem 3.1 elsewhere.

3.2 Corollary Let $M$ be a connected manifold (possibly with boundary) of dimension $\geq 3$. Let $X$ be a connected finite 2-complex with $\partial X=\emptyset$ embedded in $M$ such that the inclusion homomorphism $\pi_{1}(X) \rightarrow \pi_{1}(M)$ is an isomorphism. Let $S \subset M$ be a cooriented compact submanifold of $M$ of codimension 1 intersecting $\partial M$ along $\partial S$ and intersecting $X$ transversely along a regular graph $\Gamma=S \cap X$. Let $s \in H^{1}(M ; \mathbb{Z})$ be the cohomology class represented by $S$. Then

$$
|\chi(\Gamma)| \geq \max _{\sigma \in\left(\operatorname{Tors} H_{1}(M)\right)^{*}}\left(|| s||^{\sigma}-\delta_{\sigma}^{1}|s|_{0}\right)
$$

where $\|\ldots\|^{\sigma}$ is the Alexander-Fox norm on $H^{1}(M ; \mathbb{R})$ determined by $\sigma$. 
The assumption $\pi_{1}(X)=\pi_{1}(M)$ ensures that $\pi_{1}(M)$ is finitely generated so that the AF-norms on $H^{1}(M ; \mathbb{R})$ are well defined.

To deduce Corollary 3.2 from Theorem 3.1 , set $s^{\prime}=\left.s\right|_{X} \in H^{1}(X ; \mathbb{R})$. Clearly, $s^{\prime}=s_{\Gamma}$. Therefore $|\chi(\Gamma)|=\chi_{-}(\Gamma) \geq\left\|s^{\prime}\right\|$. By Theorem 3.1 and the assumption $\pi_{1}(X)=\pi_{1}(M)$

$$
\left\|s^{\prime}\right\| \geq \max _{\sigma \in\left(\operatorname{Tors} H_{1}(X)\right)^{*}}\left(\| s^{\prime}||^{\sigma}-\delta_{\sigma}^{1}\left|s^{\prime}\right|_{0}\right)=\max _{\sigma \in\left(\operatorname{Tors} H_{1}(M)\right)^{*}}\left(\|s\|^{\sigma}-\delta_{\sigma}^{1}|s|_{0}\right) .
$$

The statement of Corollary 3.2 is not specific about the category of manifolds. In fact the corollary extends to a much broader setting where $M$ is an arcwise connected space and $S$ is a subspace of $M$ which has a cylinder neighborhood $U=S \times[-1,1] \subset M$ such that $S=S \times 0$. A coorientation of $S$ is defined in the obvious way and determines a cohomology class $s \in H^{1}(M)$ as in Sect. 1.2. As above, $X \subset M$ is a connected finite 2-complex with $\partial X=\emptyset$ such that the inclusion homomorphism $\pi_{1}(X) \rightarrow \pi_{1}(M)$ is an isomorphism and $U \cap X=\Gamma \times[-1,1]$ where $\Gamma=S \cap X$ is a graph in $X$. Then we have (3.b).

Corollary 3.2 can be applied in various geometric situations. For instance, if $M$ is a compact triangulated manifold of dimension $\geq 3$ then we can take $X$ to be the 2-skeleton of $M$. If $M$ is a compact 3-manifold then we can take $X$ to be a spine of $M$ or a spine of punctured $M$.

3.3 Corollary Let $\pi$ be a group presented by a finite number of generators and relations $\left\langle x_{1}, \ldots, x_{m}: r_{1}, \ldots, r_{n}\right\rangle$ where $r_{1}, \ldots, r_{n}$ are words in the alphabet $x_{1}^{ \pm 1}, \ldots, x_{m}^{ \pm 1}$ such that (in the notation of Sect. 1.8) $\#\left(x_{i}\right) \geq 2$ for $i=1, \ldots, m$. Then for any $s \in H^{1}(\pi ; \mathbb{R})$,

$$
\sum_{i=1}^{n}\left(\#\left(x_{i}\right) / 2-1\right)\left|s\left(x_{i}\right)\right| \geq \max _{\sigma \in\left(\operatorname{Tors} H_{1}(\pi)\right)^{*}}\left(\left.|| s\right|^{\sigma}-\delta_{\sigma}^{1}|s|_{0}\right) .
$$

This corollary is obtained by an application of Theorem 3.1 to the 2-complex determined by the presentation $\left\langle x_{1}, \ldots, x_{m}: r_{1}, \ldots, r_{n}\right\rangle$.

3.4 Examples (1) The computations in Sect. 1.8 and 2.4.2 show that for the group presentation $\left\langle x, y: x^{p} y^{q}=1\right\rangle$ the inequality (3.c) is an equality. Thus, this presentation is minimal in the sense of Sect. 1.8. (It would be interesting to extend this fact to groups of other fibered knots in $S^{3}$ ).

(2) Let $\pi=\left\langle x, y: x^{k} y^{l} x^{-k} y^{-l}=1, y^{m}=1\right\rangle$ where $k, l \geq 1, m \geq 2$. We claim that if $m$ does not divide $l$ then this presentation is minimal. Indeed, there is 
a nontrivial character $\sigma$ of $\operatorname{Tors} H_{1}(\pi)=\mathbb{Z} / m \mathbb{Z}$ such that $(\sigma([y]))^{l} \neq 1$. The computations in Sect. 2.4.3 show that $\|s\|^{\sigma}=k-1$ where $s$ is a generator of $H^{1}(\pi)=\mathbb{Z}$. The left hand side of (3.c) is $(2 k) / 2-1=k-1$. Hence (3.c) is an equality for this presentation of $\pi$ which is therefore minimal. Examples 1 and 2 show that the estimate in Theorem 3.1 is sharp.

(3) Consider $\Gamma, f, X, s$ from the mapping torus of Example 1.7.2. We will deduce the equality $\|s\|=\chi_{-}(\Gamma)$ from Theorem 3.1. We need only to prove that $\|s\| \geq \chi_{-}(\Gamma)$. It is enough to consider the case of connected $\Gamma$. By (3.a), it is enough to show that $\chi_{-}(\Gamma)=\|s\|^{1}-|s|_{0}$. To this end we shall compute the (untwisted) AF-polynomial $\Delta(X)=\Delta\left(\pi_{1}(X)\right)$. We can deform $f: \Gamma \rightarrow \Gamma$ so that it fixes a point $\gamma \in \Gamma$. Let $x_{1}, \ldots, x_{n}$ be free generators of the free group $\pi_{1}(\Gamma, \gamma)$ where $n \geq 1$. The group $\pi_{1}(X)$ can be presented by $n+1$ generators $x_{1}, \ldots, x_{n}, T$ subject to $n$ relations $T x_{i} T^{-1}\left(f_{\#}\left(x_{i}\right)\right)^{-1}=1$ where $i=1, \ldots, n$ and $f_{\#}$ is the endomorphism of $\pi_{1}(\Gamma, \gamma)$ induced by $f$. Set $G=H_{1}(X) /$ Tors $H_{1}(X)$ and let $G^{\prime}$ be the corank 1 sublattice of $G$ generated by the classes $\left[x_{1}\right], \ldots,\left[x_{n}\right] \in G$ of $x_{1}, \ldots, x_{n}$. Set $\delta=1$ if $\operatorname{rk} G \geq 2$ and $\delta=0$ if $\operatorname{rk} G=1$. A direct computation using the Fox differential calculus gives $\Delta(X)=(t-1)^{-\delta} \operatorname{det}\left(t E_{n}-A\right)$ where $t=[T] \in G$ is the class of $T, E_{n}$ is the unit $(n \times n)$-matrix, and $A$ is the $(n \times n)$-matrix over $\mathbb{Z}\left[G^{\prime}\right]$ obtained as the image of the matrix $\left(\partial f_{\#}\left(x_{i}\right) / \partial x_{j}\right)_{i, j=1, \ldots, n}$ under the natural ring homomorphism $\mathbb{Z}\left[\pi_{1}(\Gamma, \gamma)\right] \rightarrow \mathbb{Z}\left[G^{\prime}\right]$ sending each $x_{i}$ to $\left[x_{i}\right]$. Clearly, $\operatorname{det}\left(t E_{n}-A\right)=a_{0}+a_{1} t+$ $\ldots+a_{n-1} t^{n-1}+t^{n}$ where $a_{0}, \ldots, a_{n-1} \in \mathbb{Z}\left[G^{\prime}\right]$. Since $f_{\#}$ is an isomorphism, the sum of coefficients of $a_{0}= \pm \operatorname{det} A \in \mathbb{Z}\left[G^{\prime}\right]$ is \pm 1 and therefore $a_{0} \neq 0$. By definition, $s\left(G^{\prime}\right)=0$ and $s(t)= \pm 1$. If $\operatorname{rk} G \geq 2$ then $\Delta(X)=-a_{0}+\ldots+t^{n-1}$ and $\|s\|^{1}-|s|_{0}=\|s\|^{1}=n-1=\chi_{-}(\Gamma)$. If $\operatorname{rk} G=1$ then $\Delta(X)=a_{0}+\ldots+t^{n}$ and $\|s\|^{1}-|s|_{0}=n-1=\chi_{-}(\Gamma)$.

\section{Proof of Theorem 3.1}

4.1 Preliminaries on modules Let $\Lambda$ be a commutative ring with unit. For a finitely generated $\Lambda$-module $X$ consider a $\Lambda$-linear homomorphism $f: \Lambda^{n} \rightarrow$ $\Lambda^{m}$ with finite $m$ and Coker $f=X$. The $i$-th elementary ideal $E_{i}(X) \subset \Lambda$ with $i=0,1, \ldots$ is generated by the $(m-i)$-minors of the matrix of $f$. If $m-i>n$ then $E_{i}(X)=0$; if $m-i \leq 0$, then $E_{i}(X)=\Lambda$. The ideal $E_{i}(X)$ is independent of $f$. If $\Lambda$ is a unique factorization domain then the $i$-th Alexander invariant $\Delta_{i}(X) \in \Lambda$ of $X$ is the greatest common divisor of the elements of $E_{i}(X)$. It is well-defined up to multiplication by units of $\Lambda$. Note that $\Delta_{i}(X \oplus \Lambda)=\Delta_{i-1}(X)$ for $i \geq 1$. 
Let $\Lambda=\mathbb{C}\left[t^{ \pm 1}\right]$. The Alexander invariants of a finitely generated $\Lambda$-module $X$ can be computed as follows. Since $\Lambda$ is a principal ideal domain, $X=$ $\oplus_{r=1}^{m}\left(\Lambda / \lambda_{r}\right)$ where $\lambda_{1}, \ldots, \lambda_{m} \in \Lambda$ and $\lambda_{i+1}$ divides $\lambda_{i}$ for all $i$. Then $\Delta_{i}(X)=$ $\prod_{r=i+1}^{m} \lambda_{r}$ for $i<m$ and $\Delta_{i}(X)=1$ for $i \geq m$. The maximal $r$ such that $\lambda_{1}=\ldots=\lambda_{r}=0$ is called the rank of $X$ and denoted $\operatorname{rk}_{\Lambda} X$. It is clear that $\operatorname{rk}_{\Lambda} X=\operatorname{dim}_{\mathbb{Q}(t)}\left(\mathbb{Q}(t) \otimes_{\Lambda} X\right)$ where $\mathbb{Q}(t)$ is the field of fractions of $\Lambda$. We have $\operatorname{rk}_{\Lambda} X=0 \Leftrightarrow \mathbb{Q}(t) \otimes_{\Lambda} X=0 \Leftrightarrow \Delta_{0}(X) \neq 0$. If $\operatorname{rk}_{\Lambda} X=0$ then $X$ is a finite dimensional $\mathbb{C}$-linear space and $\operatorname{dim}_{\mathbb{C}} X=\operatorname{span} \Delta_{0}(\mathrm{X})$ where the span of a nonzero Laurent polynomial $\sum_{n} a_{n} t^{n} \in \Lambda$ is $\max _{m, n, a_{m} a_{n} \neq 0}|m-n|$.

4.2 Preliminaries on twisted homology We recall the notion of twisted homology. Let $X$ be a connected $\mathrm{CW}$-space and $H=H_{1}(X)$. Let $\Lambda$ be a commutative ring with unit and $\varphi$ be a ring homomorphism $\mathbb{Z}[H] \rightarrow \Lambda$. We view $\Lambda$ as a (right) $\mathbb{Z}[H]$-module via $\lambda z=\lambda \varphi(z)$ for $\lambda \in \Lambda, z \in \mathbb{Z}[H]$. Let $p: \hat{X} \rightarrow X$ be the maximal abelian covering of $X$ (with induced CW-structure) corresponding to the commutant of $\pi_{1}(X)$. The action of $H$ on $\hat{X}$ by deck transformations makes the cellular chain complex $C_{*}(\hat{X})$ a complex of (free) left $\mathbb{Z}[H]$-modules. By definition,

$$
H_{*}^{\varphi}(X)=H_{*}\left(\Lambda \otimes_{\mathbb{Z}[H]} C_{*}(\hat{X})\right) .
$$

Note that $H_{*}^{\varphi}(X)$ is a $\Lambda$-module. The twisted homology extends to cellular pairs $Y \subset X$ by

$$
H_{*}^{\varphi}(X, Y)=H_{*}\left(\Lambda \otimes_{\mathbb{Z}[H]} C_{*}(\hat{X}) / C_{*}\left(p^{-1}(Y)\right)\right)
$$

where $C_{*}\left(p^{-1}(Y)\right)$ is the chain subcomplex of $C_{*}(\hat{X})$ generated by cells of $\hat{X}$ lying in $p^{-1}(Y)$.

The twisted homology is invariant under cellular subdivisions and forms the usual exact homology sequences such as the Mayer-Vietoris homology sequence and the homology sequence of a pair. Using a CW-decomposition of $X$ with one 0 -cell, one can check that $H_{0}^{\varphi}(X)=\Lambda / \varphi(I) \Lambda$ where $I$ is the augmentation ideal of $\mathbb{Z}[H]$.

4.3 Preliminaries on weighted graphs The notion of weighted graphs formalizes graphs with parallel components. A weighted graph in a 2-complex $X$ is a cooriented regular graph $\Gamma \subset X$ such that each its component $\Gamma_{i}$ is endowed with a positive integer $w_{i}$ called the weight of $\Gamma_{i}$. We write $\Gamma=$ $\cup_{i}\left(\Gamma_{i}, w_{i}\right)$. A weighted graph $\Gamma=\cup_{i}\left(\Gamma_{i}, w_{i}\right)$ in $X$ gives rise to an (unweighted) cooriented regular graph $\Gamma^{u} \subset X$ obtained by replacing each $\Gamma_{i}$ by $w_{i}$ parallel copies in a small neighborhood of $\Gamma_{i}$. We say that $\Gamma$ represents the cohomology class $s_{\Gamma}=s_{\Gamma^{u}}=\sum_{i} w_{i} s_{\Gamma_{i}} \in H^{1}(X)$. Set $\chi_{-}(\Gamma)=\chi_{-}\left(\Gamma^{u}\right)=\sum_{i} w_{i} \chi_{-}\left(\Gamma_{i}\right)$. 
4.4 Lemma Let $X$ be a connected finite 2-complex with $\partial X=\emptyset$. Every nonzero $s \in H^{1}(X)$ can be represented by a weighted graph $\Gamma \subset X$ such that $\chi_{-}(\Gamma)=\|s\|$ and $X \backslash \Gamma$ is connected.

Proof Consider first an arbitrary weighted graph $\Gamma=\cup_{i}\left(\Gamma_{i}, w_{i}\right)$ in $X$. By "decreasing the weight of $\Gamma_{i}$ by 1 " we mean the transformation which reduces $w_{i}$ by 1 and keeps the other weights. If $w_{i}=1$, then this transformation removes $\Gamma_{i}$ from $\Gamma$.

Assume that $X \backslash \Gamma$ is not connected. For a component $N$ of $X \backslash \Gamma$, we define a reduction of $\Gamma$ along $N$. Let $\alpha_{+}$(resp. $\alpha_{-}$) be the set of all $i$ such that $N$ is adjacent to $\Gamma_{i}$ only on the positive (resp. negative) side. The sets $\alpha_{+}, \alpha_{-}$are disjoint. Since $N \neq X \backslash \Gamma$, at least one of these two sets is non-void. Counting the number of entries and exits in $N$ of a loop on $X$ we observe that $\sum_{i \in \alpha_{+}} s_{\Gamma_{i}}=\sum_{i \in \alpha_{-}} s_{\Gamma_{i}} \in H^{1}(X)$. We modify $\Gamma$ as follows. If $\alpha_{+} \neq \emptyset$ and $\sum_{i \in \alpha_{+}} \chi_{-}\left(\Gamma_{i}\right) \geq \sum_{i \in \alpha_{-}} \chi_{-}\left(\Gamma_{i}\right)$, then we decrease by 1 the weights of all $\left\{\Gamma_{i}\right\}_{i \in \alpha_{+}}$and increase by 1 the weights of all $\left\{\Gamma_{i}\right\}_{i \in \alpha_{-}}$. If $\alpha_{+}=\emptyset$ or $\sum_{i \in \alpha_{+}} \chi_{-}\left(\Gamma_{i}\right)<\sum_{i \in \alpha_{-}} \chi_{-}\left(\Gamma_{i}\right)$ then we increase by 1 the weights of all $\left\{\Gamma_{i}\right\}_{i \in \alpha_{+}}$ and decrease by 1 the weights of all $\left\{\Gamma_{i}\right\}_{i \in \alpha_{-}}$. This yields another weighted graph $\Gamma^{\prime}$ such that $s_{\Gamma^{\prime}}=s_{\Gamma}$ and $\chi_{-}\left(\Gamma^{\prime}\right) \leq \chi_{-}(\Gamma)$. Iterating this transformation, we eventually remove from $\Gamma$ at least one component incident to $N$ on one side. Let us call this iteration the reduction of $\Gamma$ along $N$. The reduction does not increase $\chi_{-}$, preserves $s_{\Gamma}$ and strictly decreases the number of components of $X \backslash \Gamma$. If $\partial \bar{N}$ is connected then the reduction along $N$ removes $\partial \bar{N}$ from $\Gamma$.

To prove the lemma, represent $s$ by a cooriented regular graph $S \subset X$ such that $\chi_{-}(S)=\|s\|$. We view $S$ as a weighted graph with weights of all components equal to 1 . If $X \backslash S$ is connected then $S$ satisfies the requirements of the lemma. If $X \backslash S$ is not connected then iteratively applying to $S$ reductions along components of $X \backslash S$ we eventually obtain a weighted graph, $\Gamma$, such that $X \backslash \Gamma$ is connected. Clearly, $s_{\Gamma}=s$. We have $\chi_{-}(\Gamma)=\|s\|$, since

$$
\|s\| \leq \chi_{-}\left(\Gamma^{u}\right)=\chi_{-}(\Gamma) \leq \chi_{-}(S)=\|s\| \text {. }
$$

4.5 Proof of Theorem 3.1 Set $\pi=\pi_{1}(X), H=H_{1}(X), G=H / \operatorname{Tors} H$. If $\Delta^{\sigma}(\pi)=0$ then $\|s\|^{\sigma}=0$ and $\|s\|^{\sigma}-\delta_{\sigma}^{1}|s|_{0} \leq 0 \leq\|s\|$. Assume from now on that $\Delta^{\sigma}(\pi) \neq 0$.

Fix a splitting $H=$ Tors $H \times G$ and consider the ring homomorphism $\tilde{\sigma}$ : $\mathbb{Z}[H] \rightarrow \mathbb{C}[G]$ sending $f g$ with $f \in$ Tors $H, g \in G$ to $\sigma(f) g$. By assumption, $\operatorname{gcd} \tilde{\sigma}(E(\pi))=\Delta^{\sigma}(\pi) \neq 0$ so that $\tilde{\sigma}(E(\pi)) \neq 0$. Pick a representative 
$\sum_{g \in G} c_{g} g$ of $\Delta^{\sigma}(\pi)$. Pick a nonzero $\mu=\sum_{g \in G} \mu_{g} g \in \tilde{\sigma}(E(\pi))$ where $\mu_{g} \in \mathbb{C}$. We call $s \in H^{1}(X)$ regular if $s(g) \neq s\left(g^{\prime}\right)$ for any distinct $g, g^{\prime} \in G$ such that $c_{g} c_{g^{\prime}} \neq 0$ or $\mu_{g} \mu_{g^{\prime}} \neq 0$. (For $\operatorname{rk} G \geq 2$, this notion depends on the choice of $\mu$. In the case $\operatorname{rk} G=1$ all nonzero $s$ are regular). The set of regular $s$ is the complement in $H^{1}(X)$ of a finite set of sublattices of corank 1 . We call $s \in H^{1}(X)$ primitive if its evaluation on a certain element of $H$ equals 1 .

Since the norms $\|\ldots\|,\|\ldots\|^{\sigma}$, and $|\ldots|_{0}$ on $H^{1}(X ; \mathbb{R})$ are continuous and homogeneous, it suffices to prove that $\|s\| \geq\|s\|^{\sigma}-\delta_{\sigma}^{1}|s|_{0}$ for primitive regular $s \in H^{1}(X)$. Fix a primitive regular $s \in H^{1}(X)$. (In the case $\operatorname{rk} G=1, s$ is any generator of $\left.H^{1}(X)=\mathbb{Z}\right)$. Let $\varphi: \mathbb{Z}[H] \rightarrow \mathbb{C}\left[t^{ \pm 1}\right]=\Lambda$ be the composition of $\tilde{\sigma}: \mathbb{Z}[H] \rightarrow \mathbb{C}[G]$ and the $\mathbb{C}$-linear ring homomorphism $\tilde{s}: \mathbb{C}[G] \rightarrow \Lambda=\mathbb{C}\left[t^{ \pm 1}\right]$ sending any $g \in G$ to $t^{s(g)}$. Recall the $\Lambda$-module $H_{*}^{\varphi}(X)$ (see Sect. 4.2).

Claim $1 \quad \Delta_{0}\left(H_{1}^{\varphi}(X)\right) \in \Lambda$ is non-zero and divisible by $(t-1)^{\delta} \sum_{g \in G} c_{g} t^{s(g)}$ where $\delta=1$ if $\sigma=1$ and $\operatorname{rk} G \geq 2$ and $\delta=0$ otherwise.

By the regularity of $s$, the polynomial $(t-1)^{\delta} \sum_{g} c_{g} t^{s(g)}$ is nonzero and its span equals $\delta+\|s\|^{\sigma}$. Claim 1 implies that

$$
\operatorname{dim}_{\mathbb{C}} H_{1}^{\varphi}(X)=\operatorname{span} \Delta_{0}\left(H_{1}^{\varphi}(X)\right) \geq \delta+\|s\|^{\sigma}=\delta_{\sigma}^{1}\left(1-|s|_{0}\right)+\|s\|^{\sigma} .
$$

The inequality $\|s\| \geq \|\left. s\right|^{\sigma}-\delta_{\sigma}^{1}|s|_{0}$ follows now from the next claim.

Claim $2 \quad\|s\| \geq \operatorname{dim}_{\mathbb{C}} H_{1}^{\varphi}(X)-\delta_{\sigma}^{1}$.

Now we prove Claims 1 and 2 .

Proof of Claim 1 Contracting recursively the 1-cells of $X$ with distinct endpoints we obtain a finite 2-dimensional CW-complex homotopy equivalent to $X$ and having only one 0 -cell. Since $H_{1}^{\varphi}(X)$ and $\Delta^{\sigma}(\pi)$ are homotopy invariants, we can assume in the proof of Claim 1 that $X$ has only one 0 -cell, $x$. Consider the presentation of $\pi=\pi_{1}(X, x)$ determined by the cellular structure of $X$. The corresponding Alexander matrix is nothing but the matrix of the $\mathbb{Z}[H]$-linear boundary homomorphism $C_{2}(\hat{X}) \rightarrow C_{1}(\hat{X})$ where $\hat{X}$ is the maximal abelian covering of $X$. Applying $\varphi: \mathbb{Z}[H] \rightarrow \Lambda$ to the entries of this matrix we obtain a presentation matrix of the $\Lambda$-module $H_{1}^{\varphi}(X, x)$. Therefore $E_{1}\left(H_{1}^{\varphi}(X, x)\right)=\mathbb{C} \cdot \varphi(E(\pi)) \subset \Lambda$.

Recall that $\varphi=\tilde{s} \circ \tilde{\sigma}$. By definition of $\Delta^{\sigma}(\pi)$, we have $\tilde{\sigma}(E(\pi)) \subset \Delta^{\sigma}(\pi) \mathbb{C}[G]$. If $\sigma=1$ and $\operatorname{rk} G \geq 2$, then (2.a) implies a stronger inclusion $\tilde{\sigma}(E(\pi)) \subset$ $\Delta^{\sigma}(\pi) J$ where $J$ is the augmentation ideal of $\mathbb{C}[G]$. Applying $\tilde{s}$, we obtain 
that $\varphi(E(\pi))=(\tilde{s} \circ \tilde{\sigma})(E(\pi))$ is contained in the principal ideal generated by $(t-1)^{\delta} \tilde{s}\left(\Delta^{\sigma}(\pi)\right)=(t-1)^{\delta} \sum_{g} c_{g} t^{s(g)}$. The regularity of $s$ implies that $\varphi(E(\pi)) \neq 0$. Hence $\Delta_{1}\left(H_{1}^{\varphi}(X, x)\right)=\operatorname{gcd} \varphi(E(\pi))$ is non-zero and divisible by $(t-1)^{\delta} \sum_{g \in G} c_{g} t^{s(g)}$. It remains to observe that $\Delta_{0}\left(H_{1}^{\varphi}(X)\right)=\Delta_{1}\left(H_{1}^{\varphi}(X, x)\right)$. Indeed, consider the exact sequence

$$
0 \rightarrow H_{1}^{\varphi}(X) \rightarrow H_{1}^{\varphi}(X, x) \rightarrow H_{0}^{\varphi}(x) \rightarrow H_{0}^{\varphi}(X) .
$$

Clearly, $H_{0}^{\varphi}(x)=\Lambda$ and $H_{0}^{\varphi}(X)=\Lambda / \varphi(I) \Lambda$ where $I$ is the augmentation ideal of $\mathbb{Z}[H]$. The kernel $\varphi(I) \Lambda \subset \Lambda$ of the inclusion homomorphism $H_{0}^{\varphi}(x) \rightarrow$ $H_{0}^{\varphi}(X)$ is a free $\Lambda$-module of rank 1 . Hence $H_{1}^{\varphi}(X, x)=H_{1}^{\varphi}(X) \oplus \Lambda$ and $\Delta_{0}\left(H_{1}^{\varphi}(X)\right)=\Delta_{1}\left(H_{1}^{\varphi}(X, x)\right)$.

Proof of Claim 2 Consider the ring homomorphism $\bar{\sigma}=\operatorname{aug} \circ \tilde{\sigma}: \mathbb{Z}[H] \rightarrow \mathbb{C}$ mapping $G \subset H$ to 1 and mapping any $f \in \operatorname{Tors} H$ to $\sigma(f) \in \mathbb{C}^{*}$. We call a cellular set $S \subset X$ bad if $\bar{\sigma}$ is trivial on $H_{1}(S)$, i.e., if the composition of the inclusion homomorphism $H_{1}(S) \rightarrow H$ with $\bar{\sigma}$ maps $H_{1}(S)$ to 1 .

By Lemma 4.4, there is a weighted graph $\Gamma=\cup_{i}\left(\Gamma_{i}, w_{i}\right) \subset X$ such that $X \backslash \Gamma$ is connected, $s_{\Gamma}=s$, and $\chi_{-}(\Gamma)=\|s\|$. We first compute the $\varphi$-twisted homology $H_{*}^{\varphi}\left(\Gamma_{i}\right)$ of a component $\Gamma_{i}$ of $\Gamma$. Observe that $s$ annihilates $H_{1}\left(\Gamma_{i}\right)$ and therefore $\left.\varphi\right|_{H_{1}\left(\Gamma_{i}\right)}$ is the composition of $\left.\bar{\sigma}\right|_{H_{1}\left(\Gamma_{i}\right)}$ with the inclusion $\mathbb{C} \subset \Lambda$. Hence $H_{*}^{\varphi}\left(\Gamma_{i}\right)=\Lambda \otimes_{\mathbb{C}} H_{*}^{\bar{\sigma}}\left(\Gamma_{i}\right)$. If $\Gamma_{i}$ is bad then $H_{*}^{\bar{\sigma}}\left(\Gamma_{i}\right)$ is the usual untwisted homology of $\Gamma_{i}$ with complex coefficients. If $\Gamma_{i}$ is not bad then $H_{0}^{\bar{\sigma}}\left(\Gamma_{i}\right)=0$ and $\operatorname{dim}_{\mathbb{C}} H_{1}^{\bar{\sigma}}\left(\Gamma_{i}\right)=-\chi\left(\Gamma_{i}\right)=\chi_{-}\left(\Gamma_{i}\right) \geq 0$.

Let $U=\Gamma \times[-1,1]$ be a closed regular neighborhood of $\Gamma$ in $X$ such that $\Gamma=\Gamma \times 0$. We can assume that the given coorientation of $\Gamma$ is determined by $\Gamma \times(0,1] \subset U$. Set $N=\overline{X \backslash U}$. By our assumptions, $N$ is connected. Clearly, $N \cap U=\partial N=\partial U$ contains two copies $\Gamma_{i}^{ \pm}=\Gamma_{i} \times( \pm 1)$ of each $\Gamma_{i}$.

The Mayer-Vietoris homology sequence of the triple $(X=N \cup U, N, U)$, gives an exact sequence

$$
H_{1}^{\varphi}(X) \rightarrow H_{0}^{\varphi}(N \cap U) \rightarrow H_{0}^{\varphi}(N) \oplus H_{0}^{\varphi}(U) .
$$

It is clear that $s$ annihilates $H_{1}(N)$ and therefore $H_{0}^{\varphi}(N)=\Lambda \otimes_{\mathbb{C}} H_{0}^{\bar{\sigma}}(N)=\Lambda^{\beta}$ where $\beta=1$ if $N$ is bad and $\beta=0$ otherwise. The computations above show that $H_{0}^{\varphi}(U)=H_{0}^{\varphi}(\Gamma)=\Lambda^{\alpha}$ where $\alpha$ is the number of bad components of $\Gamma$. Similarly, $H_{0}^{\varphi}(N \cap U)=\Lambda^{2 \alpha}$. Therefore,

$$
0=\operatorname{rk}_{\Lambda} H_{1}^{\varphi}(X) \geq 2 \alpha-(\alpha+\beta)=\alpha-\beta .
$$

Hence $\alpha \leq \beta=0,1$. 
Case $\beta=1$ In this case $N$ is bad and therefore all its boundary components $\Gamma_{i}^{ \pm}$are bad. Thus, all the components of $\Gamma$ are bad. The inequality $\alpha \leq \beta=1$ implies that $\Gamma$ is connected. Since the dual class $s$ is primitive, the weight of (the only component of) $\Gamma$ is equal to 1 . Thus $\|s\|=\chi_{-}(\Gamma)$.

Since $\Gamma$ and its complement in $X$ are bad, the group homomorphism $\left.\bar{\sigma}\right|_{H}: H \rightarrow$ $\mathbb{C}^{*}$ is a composition of $s: H \rightarrow \mathbb{Z}$ with a certain group homomorphism $\mathbb{Z} \rightarrow \mathbb{C}^{*}$. Such a composition is trivial on Tors $H$. Hence $\sigma=1$. Then $H_{1}^{\varphi}(X)=$ $H_{1}(\tilde{X} ; \mathbb{C})$ where $\tilde{X} \rightarrow X$ is the infinite cyclic covering determined by $s$. To prove Claim 2, it suffices to prove the inequality $\chi_{-}(\Gamma) \geq \operatorname{dim}_{\mathbb{C}} H_{1}(\tilde{X} ; \mathbb{C})-1$. Observe that the graph $\Gamma$ lifts to a homeomorphic graph $\tilde{\tilde{\Gamma}} \subset \tilde{X}$ splitting $\tilde{X}$ into two connected pieces, $\tilde{X}_{-}$and $\tilde{X}_{+}$. Let $t$ be the generating deck transformation of the covering $\tilde{X} \rightarrow X$ such that $t \tilde{X}_{+} \subset \tilde{X}_{+}$. The characteristic polynomial of the action of $t$ on $H_{1}(\tilde{X}, \mathbb{C})$ is $\Delta_{0}\left(H_{1}(\tilde{X} ; \mathbb{C})\right)=\Delta_{0}\left(H_{1}^{\varphi}(X)\right) \neq 0$. Applying to any compact subset of $\tilde{X}$ a sufficiently big positive (resp. negative) power of $t$ we can translate this subset into $\tilde{X}_{+}\left(\operatorname{resp} . \tilde{X}_{-}\right)$. This implies that the inclusion homomorphisms $H_{1}\left(\tilde{X}_{-} ; \mathbb{C}\right) \rightarrow H_{1}(\tilde{X}, \mathbb{C})$ and $H_{1}\left(\tilde{X}_{+} ; \mathbb{C}\right) \rightarrow H_{1}(\tilde{X}, \mathbb{C})$ are surjective. The Mayer-Vietoris homology sequence for $\tilde{X}=\tilde{X}_{+} \cup \tilde{X}_{-}$implies the surjectivity of the inclusion homomorphism $H_{1}(\tilde{\Gamma} ; \mathbb{C}) \rightarrow H_{1}(\tilde{X}, \mathbb{C})$. Computing the dimensions, we obtain $\chi_{-}(\Gamma)+1=\operatorname{dim}_{\mathbb{C}} H_{1}(\Gamma ; \mathbb{C}) \geq \operatorname{dim}_{\mathbb{C}} H_{1}(\tilde{X} ; \mathbb{C})$.

Case $\beta=0$ In this case $N$ is not bad and $\alpha=\beta=0$ so that $\Gamma$ has no bad components. In particular, $\sigma \neq 1$.

Now we compute $H_{1}^{\varphi}(X)$. Let $\Gamma_{1}, \ldots, \Gamma_{n}$ be the components of $\Gamma$ with weights $w_{1}, \ldots, w_{n}$. As we know $H_{0}^{\varphi}(N \cap U)=\Lambda^{2 \alpha}=0$. The Mayer-Vietoris homology sequence of the triple $(X=N \cup U, N, U)$ yields that the inclusion homomorphism $H_{1}^{\varphi}(N) \rightarrow H_{1}^{\varphi}(X)$ is surjective and its kernel is generated by the vectors $i n\left(t^{w_{i}} x-f_{i}^{\varphi}(x)\right)$ where $i=1, \ldots, n ; x$ runs over $H_{1}^{\varphi}\left(\Gamma_{i}^{+}\right)$; $f_{i}^{\varphi}: H_{1}^{\varphi}\left(\Gamma_{i}^{+}\right) \rightarrow H_{1}^{\varphi}\left(\Gamma_{i}^{-}\right)$is the isomorphism induced by the natural homeomorphisms $\Gamma_{i}^{+} \approx \Gamma_{i} \approx \Gamma_{i}^{-}$; and in is the inclusion homomorphism $H_{1}^{\varphi}(\partial N) \rightarrow$ $H_{1}^{\varphi}(N)$. We claim that in is surjective. Indeed, since $s$ annihilates $H_{1}(N)$ we have $H_{1}^{\varphi}(N)=\Lambda \otimes_{\mathbb{C}} H_{1}^{\bar{\sigma}}(N)$ and $H_{1}^{\varphi}(\partial N)=\Lambda \otimes_{\mathbb{C}} H_{1}^{\bar{\sigma}}(\partial N)$. Moreover, in $=\operatorname{id}_{\Lambda} \otimes_{\mathbb{C}} j$ where $j: H_{1}^{\bar{\sigma}}(\partial N) \rightarrow H_{1}^{\bar{\sigma}}(N)$ is the inclusion homomorphism. If $j$ is not surjective then the cokernel of $i n$ is a free $\Lambda$-module of rank $\geq 1$. On the other hand, this cokernel is a quotient of the finite dimensional $\mathbb{C}$-linear space $H_{1}^{\varphi}(X)$. This contradiction shows that both $j$ and in must be surjective. Therefore $H_{1}^{\varphi}(X)$ is the quotient of

$$
H_{1}^{\varphi}(\partial N)=\bigoplus_{i=1}^{n}\left(H_{1}^{\varphi}\left(\Gamma_{i}^{+}\right) \oplus H_{1}^{\varphi}\left(\Gamma_{i}^{-}\right)\right)=\Lambda \otimes_{\mathbb{C}} \bigoplus_{i=1}^{n}\left(H_{1}^{\bar{\sigma}}\left(\Gamma_{i}^{+}\right) \oplus H_{1}^{\bar{\sigma}}\left(\Gamma_{i}^{-}\right)\right)
$$


by Ker $i n=\Lambda \otimes_{\mathbb{C}} \operatorname{Ker} j$ and the vectors $t^{w_{i}} x-f_{i}^{\bar{\sigma}}(x)$ where $i$ runs over $1, \ldots, n ; x$ runs over $H_{1}^{\bar{\sigma}}\left(\Gamma_{i}^{+}\right) ; f_{i}^{\bar{\sigma}}: H_{1}^{\bar{\sigma}}\left(\Gamma_{i}^{+}\right) \rightarrow H_{1}^{\bar{\sigma}}\left(\Gamma_{i}^{-}\right)$is the isomorphism induced by the homeomorphism $\Gamma_{i}^{+} \approx \Gamma_{i}^{-}$. Hence $H_{1}^{\varphi}(X)$ is a quotient of $\Lambda \otimes_{\mathbb{C}}\left(\oplus_{i} H_{1}^{\bar{\sigma}}\left(\Gamma_{i}^{+}\right)\right)$ by vectors of type $x_{1}+\ldots+x_{n}+t^{w_{1}} y_{1}+\ldots+t^{w_{n}} y_{n}$ where $x_{i}, y_{i} \in H_{1}^{\bar{\sigma}}\left(\Gamma_{i}^{+}\right)$for all $i$. Consider the corresponding presentation matrix of $H_{1}^{\varphi}(X)$ with respect to certain bases in the $\mathbb{C}$-linear spaces $H_{1}^{\bar{\sigma}}\left(\Gamma_{1}^{+}\right), \ldots, H_{1}^{\bar{\sigma}}\left(\Gamma_{n}^{+}\right)$. The column of this matrix corresponding to any basis vector in $H_{1}^{\bar{\sigma}}\left(\Gamma_{i}^{+}\right)$has entries of type $a+t^{w_{i}} b$ with $a, b \in \mathbb{C}$. Therefore the ideal $E_{0}\left(H_{1}^{\varphi}(X)\right)$ is generated by Laurent polynomials whose span does not exceed

$$
\sum_{i=1}^{n} w_{i} \operatorname{dim}_{\mathbb{C}} H_{1}^{\bar{\sigma}}\left(\Gamma_{i}^{+}\right)=\sum_{i=1}^{n} w_{i} \chi_{-}\left(\Gamma_{i}^{+}\right)=\chi_{-}(\Gamma)=\|s\| .
$$

Therefore

$$
\operatorname{dim}_{\mathbb{C}} H_{1}^{\varphi}(X)-\delta_{\sigma}^{1}=\operatorname{dim}_{\mathbb{C}} H_{1}^{\varphi}(X)=\operatorname{span} \Delta_{0}\left(H_{1}^{\varphi}(X)\right) \leq\|s\| .
$$

\section{References}

[1] D Auckly, The Thurston norm and three-dimensional Seiberg-Witten theory, Osaka J. Math. 33 (1996) 737-750.

[2] $\mathbf{R}$ Fox, Free differential calculus. II. The isomorphism problem of groups, Ann. of Math. (2) 59 (1954) 196-210.

[3] P Kronheimer, Minimal genus in $S^{1} \times M^{3}$, Invent. Math. 135 (1999) 45-61.

[4] $\mathbf{P}$ Kronheimer, T Mrowka, The genus of embedded surfaces in the projective plane, Math. Res. Lett. 1 (1994) 797-808.

[5] P Kronheimer, T Mrowka, Scalar curvature and the Thurston norm, Math. Res. Lett. 4 (1997) 931-937.

[6] C McMullen, The Alexander polynomial of a 3-manifold and the Thurston norm on cohomology, Preprint (1998).

[7] W Thurston, A norm for the homology of 3-manifolds, Mem. Amer. Math. Soc. 59 (1986), no. 339, 99-130.

[8] V Turaev, Torsion invariants of Spin ${ }^{c}$-structures on 3-manifolds, Math. Research Letters 4 (1997) 679-695.

[9] V Turaev, Introduction to Combinatorial Torsions. Notes taken by Felix Schlenk, Lectures in Mathematics ETH Zürich. Birkhäuser Verlag, Basel (2001).

IRMA, Université Louis Pasteur - CNRS

7 rue René Descartes, 67084 Strasbourg, France

Email: turaev@math.u-strasbg.fr

Received: 1 October 2001 